\title{
Relación entre rasgos desadaptativos de personalidad y farmacodependencia
}

\author{
Cynthia De Macedo Nevenschwander
}

El presente estudio tuvo como propósito evaluar la relación entre los rasgos desadaptativos de la personalidad y la dependencia de sustancias psicoactivas en una muestra de pacientes farmacodependientes. Los instrumentos utilizados fueron el Inventario Clínico Multiaxial de Millon-II y la ficha de datos personales. Se calculó la confiabilidad en nuestro medio (0.78). La muestra, no probabilísticaaccidental, estuvo conformada por 108 participantes dependientes de sustancias psicoactivas (alcohol, marihuana, clorhidrato de cocaína, pasta básica de cocaína $y$ otras drogas) que en el momento de la evaluación se encontraban en tratamiento en tres comunidades terapéuticas y un centro de rehabilitación para adictos de Lima metropolitana. Los resultados revelan una elevada prevalencia de rasgos desadaptativos de personalidad entre los participantes del estudio.

Rasgos desadaptativos de personalidad / farmacodependencia / MCMI-II

\section{Relationship between disadaptive personality characteristics and pharmaco-dependency}

The purpose of this study was to examine the relation between personality disadaptive characteristics and dependency of psychoactive substances in a sample of pharmacodependent patients. The following instruments were used: Millon's Multiaxial Clinical Inventory II and Personal Data ficha. Confiability in our milieu was calculated. The non-probabilistic accidental sample consisted of 108 participants, who were dependent on psychoactive substances (alcohol, marihuana, cocaine hydrochloride, freebase cocaine, and other drugs). At the time of this study the participants were being treated in three therapeutic communities and a rehabilitation center for addicts in metropolitan Lima. Results show a high prevalence of disadaptive personality characteristics among participants.

disadaptive personality characteristics / pharmacodependent / MCMI-II 
Si bien el uso de sustancias psicoactivas es un fenómeno social cuyas raíces se encuentran en la historia de la humanidad, la problemática que plantea la farmacodependencia es reciente y responde a un perfeccionamiento en el empleo de estas sustancias y la elaboración de nuevas drogas sintéticas.

Según la Organización Mundial de la Salud, la farmacodependencia "es un estado de intoxicación periódico o crónico, nocivo para el individuo y la sociedad, provocado por el consumo repetido o compulsivo de un fármaco natural o sintético" (OMS, página web). Hoy en día se reconoce que la característica esencial de toda dependencia o adicción es el conjunto de signos y síntomas cognitivos, afectivos, conductuales y fisiológicos que revelan un patrón de uso compulsivo de una o varias sustancias y una incapacidad para controlar este consumo a pesar de las consecuencias negativas que conlleva para la salud del individuo y la sociedad (Oliver, 2004; Cruz, 2001).

Las explicaciones iniciales sobre el problema de la farmacodependencia atribuían el fenómeno a una causa única; estas explicaciones no encontraron mucho sustento, pues no concordaban con la naturaleza tan compleja del ser humano, por lo que dieron paso a otros enfoques que consideran que en el proceso de adquisición, desarrollo y mantenimiento de la farmacodependencia intervienen factores intrapersonales, sociales, económicos y farmacológicos
(Tapia, 2001; Ortiz, 1992; Villanueva, 1989). De hecho, diversos autores coinciden en que la farmacodependencia es un fenómeno biopsicosocial que adopta características propias en cada país, y de forma particular en cada persona, que definen su curso de acción.

Últimamente, una de las áreas que genera gran interés entre los investigadores es el estudio de las características de personalidad en los individuos dependientes de sustancias psicoactivas. Las primeras aproximaciones al estudio de la personalidad de estos sujetos intentaban delimitar o definir la existencia de una personalidad adictiva; sin embargo, hoy en día la mayoría de autores sostiene que no existe una personalidad típica, sino rasgos comunes a todos los farmacodependientes (Sánchez-Hervás \& Verjano, 1996; Lahuerta \& Lloret, 1994).

Las variables de personalidad que se han relacionado con el consumo de drogas, de acuerdo con Zavaleta (2001), son: búsqueda de sensaciones, conflicto de identidad, poca tolerancia a la frustración, rasgos antisociales, impulsividad, autoestima y autoconcepto inadecuados, sentimientos de culpa, miedo o temores irracionales, tensión, ansiedad asociada a sentimientos de inferioridad e inadecuación, entre otros. Bandura (1997) también señala que una inadecuada o pobre percepción de las propias capacidades para manejar y controlar diferentes situaciones, especialmente en la adolescen- 
cia, es un factor de riesgo para el inicio en el consumo de sustancias psicoactivas.

Por lo general, las diversas teorías coinciden en que una estructura de personalidad vulnerable constituye un factor predisponente para la dependencia a sustancias psicoactivas. Según De Casarino De Leonetti (1992), para desarrollar la farmacodependencia es necesario la confluencia de tres factores: la droga, el medio facilitador o medio social y una persona con una estructura de personalidad vulnerable. Esta vulnerabilidad, como refieren, es producto de crisis vitales o psicológicas que pueden presentarse a lo largo del desarrollo del sujeto, principalmente durante la infancia.

Sánchez-Hervás, Morales y Gradolí (2004) indican que en algunos casos puede observarse una serie de problemas previos a la farmacodependencia, relacionados con patrones rígidos $\mathrm{y}$ desadaptativos de personalidad, donde el consumo de drogas es una opción probable. Zavaleta, por su parte, afirma que para aquellos sujetos que presentan personalidades complejas o patológicas, el consumo de sustancias psicoactivas "constituye a menudo la única alternativa para enfrentarse a los problemas personales (...); estas personas suelen ser más susceptibles a reaccionar favorablemente a la adicción" (2001: 54).

Otros autores señalan que el consumo acentúa y refuerza ciertos rasgos de personalidad, que en un inicio no eran necesariamente desadaptativos. Así, muchas personas ven acentuados rasgos compulsivos, narcisistas, impulsivos, entre otros, que luego con el consumo prolongado de sustancias, pueden devenir en trastornos de personalidad si constituyen patrones inflexibles y desadaptativos de formas de percibir, pensar y relacionarse, causando malestar subjetivo y un deterioro significativo en las diversas áreas de funcionamiento del individuo.

Como se desprende de todo lo anterior, el campo de la farmacodependencia es muy complejo y delicado, siendo muy importante el estudio de las características de personalidad de los consumidores de drogas por su repercusión en el curso de dicho fenómeno.

\section{Objetivos}

\section{Objetivo general}

Estudiar la relación entre los rasgos desadaptativos de la personalidad, evaluados a través del Inventario Clínico Multiaxial de Millon-II y la dependencia a sustancias psicoactivas en una muestra de pacientes farmacodependientes de tres comunidades terapéuticas y un centro de rehabilitación para adictos en Lima metropolitana.

\section{Objetivos específicos}

- Determinar la prevalencia de consumo en la población estudiada. 
- Determinar la prevalencia de rasgos desadaptativos de personalidad entre los farmacodependientes que componen la muestra.

- Establecer la relación que existe entre los rasgos desadaptativos de personalidad y la dependencia a sustancias psicoactivas.

- Establecer si existen diferencias significativas en la dependencia a sustancias psicoactivas según edad y grado de instrucción.

- Identificar si existen diferencias de los rasgos desadaptativos de personalidad según edad, grado de instrucción, tiempo de consumo y tiempo de tratamiento.

- Evaluar si se presentan diferencias significativas de los rasgos desadaptativos de personalidad según tipo de sustancia consumida.

\section{MÉtodo}

\section{Muestra}

La muestra a la que hace referencia el presente estudio, la componen sujetos dependientes de sustancias psicoactivas (alcohol, marihuana, clorhidrato de cocaína, pasta básica de cocaína y otras drogas), que en el momento de la evaluación se encontraban en tratamiento en tres comunidades terapéuticas y en un centro de rehabilitación en Lima metropolitana.
Dada la naturaleza de la investigación, la muestra se obtuvo de forma no probabilística-accidental, ya que la elección de esta dependió de la accesibilidad y disponibilidad de los centros y sujetos entrevistados (Hernández et al., 2003).

Los criterios de inclusión a la muestra fueron:

- hablar fluidamente el idioma español;

- saber leer y escribir;

- ser dependiente de sustancias psicoactivas;

- encontrarse involucrado en un programa de salud mental o evaluación profesional, consejo o tratamiento;

- responder más del 90\% de los ítems del MCMI-II;

- obtener un puntaje menor a 1 en la escala de validez (V) del MCMI-II; $\mathrm{y}$,

- presentar un puntaje directo entre 145 y 590 en la escala de sinceridad (X).

De los 130 sujetos evaluados inicialmente, 22 fueron eliminados porque no cumplieron con los criterios de validez y sinceridad de la prueba. De este modo, la muestra válida para el análisis de datos la conforman 108 sujetos: 5 mujeres $(4,6 \%)$ y 103 hombres $(95,4 \%)$, dependientes de sustancias psicoactivas, con edades comprendidas entre los 15 y 59 años, siendo que $34,3 \%$ tienen entre 20 y 29 años. La 
Tabla 1

Composición de la muestra válida según características demográficas y clínicas

\begin{tabular}{|c|c|c|c|}
\hline Variables & Categoría & Frecuencia & $\%$ \\
\hline Sexo & $\begin{array}{l}\text { Masculino } \\
\text { Femenino }\end{array}$ & $\begin{array}{c}103 \\
5\end{array}$ & $\begin{array}{c}95,4 \\
4,6\end{array}$ \\
\hline Edad & $\begin{array}{l}15 \text { y } 19 \text { años } \\
20-29 \\
30-39 \\
40-49 \\
50-59 \text { años }\end{array}$ & $\begin{array}{l}11 \\
37 \\
23 \\
25 \\
12\end{array}$ & $\begin{array}{l}10,2 \\
34,3 \\
21,3 \\
23,1 \\
11,1\end{array}$ \\
\hline Estado civil & $\begin{array}{l}\text { Soltero } \\
\text { Casado } \\
\text { Separado/divorciado } \\
\text { Viudo }\end{array}$ & $\begin{array}{c}65 \\
19 \\
23 \\
1\end{array}$ & $\begin{array}{r}60,2 \\
17,6 \\
21,3 \\
, 9\end{array}$ \\
\hline Grado de instrucción & $\begin{array}{l}\text { Primaria } \\
\text { Secundaria } \\
\text { Superior }\end{array}$ & $\begin{array}{c}2 \\
35 \\
71\end{array}$ & $\begin{array}{r}1,8 \\
32,4 \\
65,7\end{array}$ \\
\hline Tipo de tratamiento & $\begin{array}{l}\text { Ambulatorio } \\
\text { Internamiento } \\
\text { Sin tratamiento }\end{array}$ & $\begin{array}{c}18 \\
89 \\
1\end{array}$ & $\begin{array}{r}16,7 \\
82,4 \\
, 9\end{array}$ \\
\hline Tiempo de tratamiento & $\begin{array}{l}<\text { de } 1 \text { mes } \\
1-3 \\
4-8 \\
\text { Más de } 9 \text { meses } \\
\text { Sin tratamiento }\end{array}$ & $\begin{array}{c}12 \\
47 \\
38 \\
10 \\
1\end{array}$ & $\begin{array}{r}11,1 \\
43,5 \\
35,2 \\
9,3 \\
, 9\end{array}$ \\
\hline Tiempo de consumo & $\begin{array}{l}<\text { de } 1 \text { año } \\
1 \text { a } 5 \\
6 \text { a } 10 \\
11 \text { a } 15 \\
\text { Más de } 16 \text { años }\end{array}$ & $\begin{array}{l}7 \\
44 \\
23 \\
14 \\
20\end{array}$ & $\begin{array}{r}6,5 \\
40,7 \\
21,3 \\
13,0 \\
18,5\end{array}$ \\
\hline Recaídas & $\begin{array}{l}\text { Sí } \\
\text { No }\end{array}$ & $\begin{array}{l}80 \\
28\end{array}$ & $\begin{array}{l}74,1 \\
25,9\end{array}$ \\
\hline
\end{tabular}

mayoría son solteros $(60,2 \%), 21,3 \%$ separados o divorciados y $17,6 \%$ casados. En relación al grado de instrucción, más de la mitad de la muestra $(65,7 \%)$ tiene educación superior $\mathrm{y}$ $32,4 \%$ educación secundaria. El tiempo promedio de consumo es de siete años; 74,1\% había sufrido recaídas, frente a un $25,9 \%$ que no. Casi la tota- lidad de sujetos $(99,1 \%)$, salvo uno, se encontraban en tratamiento en el momento de la evaluación; de estos, $82,4 \%$ estaban internados y $16,7 \%$ en régimen de tratamiento ambulatorio. En cuanto al tiempo de tratamiento, $11,1 \%$ llevaban internados menos de un mes; 43,5\% entre uno y tres meses y 44,5\% más de 4 meses (tabla 1 ). 


\section{Instrumentos}

Se utilizaron dos instrumentos: el Inventario Clínico Multiaxial de MillonII (MCMI-II) para evaluar los rasgos desadaptativos de personalidad, y la ficha de datos personales (véase anexo).

\section{MCMI - II}

El Inventario Clínico Multiaxial de Millon-II (MCMI-II), fue diseñado para ser un instrumento eficiente en la evaluación de un número importante de rasgos desadaptativos de personalidad y síndromes clínicos. Consta de 175 ítems, con formato de respuesta dicotómica (verdadero-falso), distribuidos en 22 escalas: 13 escalas de personalidad y 9 escalas de síndromes clínicos. Adicionalmente, cuenta con 4 escalas de control: Validez, Sinceridad, Deseabilidad y Alteración.

El MCMI-II diferencia 10 escalas básicas de personalidad que describen niveles leves de gravedad (esquizoide, evitativo, dependiente, histriónico, narcisista, antisocial, agresivo-sádico, compulsivo, pasivo-agresivo y autoderrotista/masoquista), y tres escalas de personalidad patológica más severa, que reflejan niveles moderados o marcados de deterioro (esquizotípica, borderline y paranoide). Las distintas escalas intentan evidenciar, de forma confiable, aquellos atributos o rasgos más duraderos y profundos de la personalidad que subyacen a las dificultades personales e interpersonales; distinguen el estilo de rasgos característicos de la estructura básica de personalidad y el nivel de mayor patología de aquellas estructuras.

En el presente estudio se aplicó la versión adaptada a la población española, editada por TEA en el 2002.

\section{Confiabilidad}

El método empleado para determinar la confiabilidad, en el caso de la adaptación española, fue la equivalencia racional. Este método sirve para evaluar la consistencia interna de un instrumento; es decir, el grado de correlación entre todos los reactivos de una escala (Cohen \& Swerdlik, 2001).

Los índices de confiabilidad que ofrece la prueba, a partir del estudio con una muestra clínica variada de 710 sujetos, son bastante aceptables. Para el caso de las escalas de personalidad con el grupo normativo español, se ofrece una estimación de estabilidad máxima de 0.90 y mínima de 0.63 , con promedios en torno a 0.77 .

A fin de establecer la confiabilidad de la evaluación en nuestro medio, se procedió a calcular la confiabilidad generalizada de la consistencia interna con los datos de los participantes del estudio. Esta alcanzó un valor de 0.78 , lo que permite concluir que el instrumento es confiable y por lo tanto se puede aplicar en la investigación. 


\section{Validez}

El proceso de validación del MCMI-II se dio a lo largo de todo el desarrollo de este; en la adaptación española se revisó la validez de constructo. El MCMI-II se construyó de acuerdo con un modelo estructural politético que acentúa la consistencia interna de la escala pero no requiere la independencia de las distintas escalas que caracterizan a los enfoques factoriales. De acuerdo con este modelo, las escalas del inventario deberían poseer un alto nivel de consistencia interna, aunque, a la vez, deberían evidenciar una superposición selectiva $\mathrm{y}$ un alto grado de correlación con otras escalas teóricamente relacionadas.

A pesar de la naturaleza no factorial del MCMI-II, se analizó factorialmente el instrumento, obteniendo un total de cuatro factores que explican aproximadamente un $86 \%$ de la varianza total.

Otro indicador de la utilidad y validez del MCMI-II provino de la correlación entre las escalas de este instrumento y otras escalas comparables incluidas en otras pruebas diagnósticas. La evidencia de este tipo proporciona algunos índices de validez convergente de las escalas que son medidas para ver si sus resultados son similares con escalas aparentemente comparables.

Según señalan Cohen y Swerdlik (2001), hay validez convergente si las puntuaciones en la prueba sometida a validación de constructo tienden a correlacionarse altamente en la dirección predicha con las puntuaciones en pruebas ya validadas diseñadas para medir el mismo constructo o uno similar relacionado.

La evidencia convergente en este caso provino de la correlación del MCMI-II y el consolidado MMPI-2 (Inventario Multifásico de Personalidad de Minnesota-2). Según Wiese (en Millon, 2002), los estudios al respecto evidencian una convergencia significativa, con una correlación media de 0.54 entre las escalas de personalidad de ambos inventarios. Este resultado es importante porque refleja que el MCMI-II mide lo que pretende medir $\mathrm{y}$, aunque comparte muchos aspectos con el MMPI-2, respecto a las escalas de personalidad, se trata de dos medidas claramente distintas, únicas.

\section{Procedimientos}

La administración de los instrumentos se dio entre abril y agosto del 2005. El MCMI-II se aplicó en forma colectiva, mientras que la ficha de datos personales se resolvió de forma individual o revisando las historias clínicas de cada sujeto. En promedio, las evaluaciones duraron entre 25 y 45 minutos.

Para poder llevar a cabo el análisis de las características psicométricas de la muestra, se procedió a calcular la confiabilidad generalizada de la consistencia interna del MCMI-II con los datos de los participantes del estudio. 
El análisis de las puntuaciones directas determinó que la prueba se podía utilizar de manera consistente.

Se aplicó la prueba de KolmogorovSmirnov, determinándose que existe una adecuada aproximación de las puntuaciones a la curva normal. En seguida se pasó al análisis de los estadísticos paramétricos y, finalmente, a la interpretación de los resultados.

\section{Resultados}

\section{Prevalencia del consumo}

\section{Droga de inicio}

En lo que se refiere al inicio del consumo, el $86,1 \%$ de farmacodependientes evaluados se inició con una droga legal ( $49,1 \%$ con alcohol y $37 \%$ con tabaco), mientras que el resto $(13,9 \%)$ lo hizo con drogas ilegales, entre las que la marihuana alcanzó el mayor porcentaje $(7,4 \%)$.

La tabla 2 indica que la edad de inicio promedio para las drogas sociales es entre los 10 y 14 años respecto al inicio con marihuana, que por lo general ocurre alrededor de los 15 a 19 años. Adicionalmente, notamos que $13,9 \%$ inicia su consumo antes de los 10 años.

\section{Droga principal}

En cuanto a la sustancia principal de consumo, prevalecen las drogas cocaínicas (59,3\%): 38,9\% son dependientes del clorhidrato de cocaína y $20,4 \%$ de la pasta básica de cocaína o PBC. Le siguen a estas el consumo de marihuana $(18,5 \%)$ y luego el de alcohol $(15,7 \%)$ (véase gráfico 1$)$.

Tabla 2

Prevalencia de la droga de inicio según la edad

\begin{tabular}{|c|c|c|c|c|c|}
\hline \multirow[b]{2}{*}{ Droga de inicio } & \multicolumn{4}{|c|}{ Edad de inicio } & \multirow[t]{2}{*}{ Total } \\
\hline & $\begin{array}{c}\text { Menos de } 10 \\
\text { años }\end{array}$ & $\begin{array}{c}10 \text { a } 14 \\
\text { años }\end{array}$ & $\begin{array}{c}15 \text { a } 19 \\
\text { años }\end{array}$ & $\begin{array}{c}20 \text { a más } \\
\text { años }\end{array}$ & \\
\hline \multicolumn{6}{|c|}{ Drogas legales } \\
\hline Tabaco & 7 & 25 & 8 & 0 & 40 \\
\hline Alcohol & 8 & 27 & 14 & 4 & 53 \\
\hline \multicolumn{6}{|c|}{ Drogas ilegales } \\
\hline Marihuana & 0 & 3 & 5 & 0 & 8 \\
\hline Otras drogas & 0 & 5 & 1 & 1 & 7 \\
\hline Total & 15 & 60 & 28 & 5 & 108 \\
\hline$\%$ & 13,9 & 55,5 & 26,0 & 4,6 & 100,0 \\
\hline
\end{tabular}




\section{Gráfico 1 \\ Prevalencia de consumo}

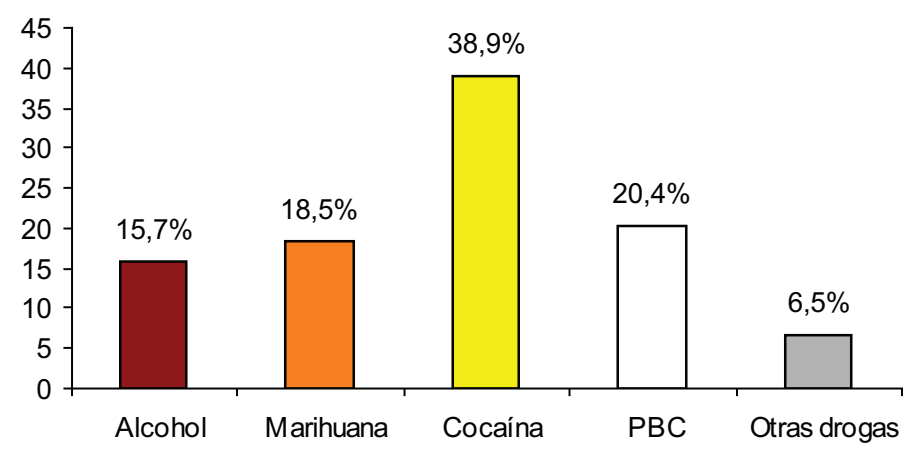

Observamos que existe relación entre el tipo de sustancia consumida y la edad (tabla 3). El alcohol tiende a consumirse entre los farmacodependientes de mayor edad, $70,6 \%$ tienen entre 40 y 59 años; la marihuana entre los de 20 a 29 años (70\%) y el clorhidrato de cocaína entre los de 30 a 49 años $(66,7 \%)$. El consumo de pasta básica de cocaína (PBC) presenta una distribución abierta.
Los hallazgos indican que existen diferencias significativas en relación con el tipo de sustancia consumida y el grado de instrucción $\left(X^{2}=8.75\right.$, $\mathrm{p}=.033$ ), advirtiéndose que los sujetos con un nivel de instrucción superior, presentan mayor consumo de clorhidrato de cocaína y marihuana respecto a los sujetos con educación secundaria.

Tabla 3

Relación entre farmacodependencia y edad

\begin{tabular}{lccccc}
\hline & \multicolumn{4}{c}{ Droga } & \\
\cline { 2 - 5 } & Alcohol & Marihuana & Cocaína & PBC & Total \\
\hline Edad & 0 & 5 & 1 & 3 & \\
15 a 19 & 2 & 14 & 10 & 8 & 9 \\
20 a 29 & 3 & 0 & 15 & 3 & 21 \\
30 a 39 & 6 & 0 & 13 & 6 & 25 \\
40 a 49 & 6 & 1 & 3 & 2 & 12 \\
50 a 59 & 17 & 20 & 42 & 22 & 101 \\
Total & & & & & \\
\hline
\end{tabular}

$\mathrm{X}^{2}=46.69$

$\mathrm{GL}=12$

$P=.000$ 
Rasgos desadaptativos de personalidad

Existe relación entre los rasgos desadaptativos de personalidad y la dependencia a sustancias psicoactivas. El $99,1 \%$ de farmacodependientes presentan por lo menos un rasgo desadaptativo de personalidad.

Se encontró una elevada prevalencia de rasgos desadaptativos de personalidad entre los farmacodependientes; el promedio es de seis rasgos desadaptativos, el $65 \%$ presentan seis o más.
Los rasgos desadaptativos de mayor prevalencia en el grupo de farmacodependientes son: compulsivo $(81,5 \%)$, antisocial $(78,7 \%)$, narcisista $(76,9 \%)$, paranoide $(72,2 \%)$, histriónico $(71,3 \%)$ y agresivo-sádico (70,4\%) (véase gráfico 2).

No se encontraron diferencias significativas de personalidad según el tipo de sustancia consumida, pudiéndose concluir que el perfil de personalidad de los farmacodependientes de esta muestra es similar (tabla 4).

\section{Gráfico 2}

Prevalencia de farmacodependientes con puntuaciones significativas en los distintos rasgos desadaptativos de personalidad del MCMI-II 
Tabla 4

Diferencias de los rasgos de personalidad según tipo de sustancia consumida

\begin{tabular}{lcccccc}
\hline Rasgos & $\begin{array}{c}\text { Alcohol } \\
\mathrm{N}=17\end{array}$ & $\begin{array}{c}\text { Marihuana } \\
\mathrm{N}=20\end{array}$ & $\begin{array}{c}\text { Cocaína } \\
\mathrm{N}=42\end{array}$ & $\begin{array}{c}\text { PBC } \\
\mathrm{N}=22\end{array}$ & $\mathrm{~F}$ & $\mathrm{p}$ \\
& $\overline{\mathrm{X}}$ & $\overline{\mathrm{X}}$ & $\overline{\mathrm{X}}$ & $\overline{\mathrm{X}}$ & & \\
\hline Esquizoide & 26.18 & 21.05 & 21.12 & 23.09 & 2.24 & .089 \\
Dependiente & 31.41 & 31.65 & 28.79 & 31.64 & 1.32 & .271 \\
Histriónico & 36.12 & 42.55 & 37.45 & 35.82 & 1.81 & .151 \\
Narcisista & 43.71 & 43.75 & 43.67 & 38.50 & 1.25 & .296 \\
Antisocial & 40.35 & 43.40 & 38.95 & 39.41 & .47 & .704 \\
Agresivo-sádico & 41.12 & 39.95 & 38.02 & 37.45 & .44 & .724 \\
Compulsivo & 42.24 & 38.45 & 40.26 & 38.41 & 1.47 & .227 \\
Esquizotípico & 24.29 & 25.75 & 19.10 & 23.86 & 1.87 & .140 \\
Borderlímite & 38.35 & 40.45 & 33.00 & 38.23 & 1.24 & .300 \\
Paranoide & 36.06 & 38.95 & 33.31 & 32.05 & 1.64 & .185 \\
\hline
\end{tabular}

Existen diferencias significativas en algunos rasgos desadaptativos de personalidad según la edad. Entre los sujetos de mayor edad se acentúan los rasgos esquizoide $(\mathrm{F}=3.43, \mathrm{p}<0.05) \mathrm{y}$ compulsivo $(\mathrm{F}=3.89, \mathrm{p}=0.006)$, mientras que entre los más jóvenes se acentúan los rasgos histriónico ( $\mathrm{F}=$ $4.77, \mathrm{p}=0.001)$ y antisocial $(\mathrm{F}=2.93$, $\mathrm{p}<0.05)$.
Hay una correlación significativa entre la edad y los rasgos de personalidad según el tipo de sustancia consumida (tabla 5). En general, podríamos concluir que los farmacodependientes de menor edad tienden a presentar rasgos desadaptativos de personalidad de forma más acentuada que los adultos.

Tabla 5

Relación entre rasgos de personalidad y edad según el tipo de sustancia consumida

\begin{tabular}{|c|c|c|c|c|c|}
\hline Grupo & & $\begin{array}{c}\text { Narcisista } \\
r\end{array}$ & $\begin{array}{c}\text { Histriónico } \\
r\end{array}$ & $\begin{array}{c}\text { Antisocial } \\
r\end{array}$ & $\begin{array}{c}\text { Paranoide } \\
r\end{array}$ \\
\hline Alcohol & $\begin{array}{l}\text { Edad } \\
p \\
N\end{array}$ & $\begin{array}{c}-.544 \\
.024 \\
17\end{array}$ & & & \\
\hline Marihuana & $\begin{array}{l}\text { Edad } \\
\mathrm{p} \\
\mathrm{N} \\
\text { Fdad }\end{array}$ & & $\begin{array}{l}-.588 \\
.006 \\
20\end{array}$ & $\begin{array}{c}-.725 \\
.000 \\
20\end{array}$ & $\begin{array}{c}-.541 \\
.014 \\
20\end{array}$ \\
\hline Cocaína & $\mathrm{p}$ & & & & \\
\hline PBC & $\begin{array}{l}\text { Edad } \\
p \\
N\end{array}$ & & & $\begin{array}{c}-.481 \\
.023 \\
22\end{array}$ & \\
\hline
\end{tabular}


Asimismo, se encontraron diferencias significativas en algunos rasgos desadaptativos de personalidad según el grado de instrucción. Los sujetos con un nivel de instrucción secundaria presentan de forma más acentuada rasgos excéntricos (esquizoides y esquizotípicos) respecto a los sujetos con educación superior. Existen diferencias en el rasgo esquizoide, por cuanto se obtiene una puntuación $\mathrm{Z}=2.47 \mathrm{y}$ una $\mathrm{p}<0.05$. De igual manera, se presentan diferencias significativas en el rasgo esquizotípico, pues se obtiene una $\mathrm{Z}=$ 2.26 y una $\mathrm{p}<0.05$.

\section{DiscusióN}

El análisis de los resultados del estudio, a la luz de la composición de la muestra, nos lleva a plantear que el consumo de drogas en forma compulsiva durante un periodo de tiempo prolongado compromete seriamente el desarrollo integral de las personas, especialmente si este se presenta o inicia en la adolescencia. La adolescencia es una etapa de transición y consolidación de la personalidad, en la cual el sujeto se enfrenta a una serie de interrogantes y dificultades que puede tratar de solucionar con la droga si es que no cuenta con la fortaleza personal ni con el apoyo externo necesario. De hecho, encontramos que la mayoría de farmacodependientes de esta muestra inició su consumo en la adolescencia y presentan una elevada prevalencia de rasgos desadaptativos de personalidad. A ello se agrega que estos rasgos estarían acentuados en los sujetos de menor edad, lo que coincide con el grupo de edad más numeroso de la muestra (20 a 29 años).

Otras consecuencias del consumo se aprecian en el área laboral y personal: 40,7\% de farmacodependientes no han completado su educación superior, $21,3 \%$ son separados o divorciados y un $60,2 \%$ son solteros. La dependencia conlleva una problemática social que podría relacionarse con el hecho de que el consumo de drogas prolonga la inmadurez y la dependencia. Se trataría de sujetos con una gran dificultad para establecer y mantener vínculos cercanos, puesto que solo saben relacionarse con un objeto: la droga; creen ser independientes pero en el fondo dependen de sus impulsos, necesidades y carencias. En este sentido, Pelicier se refiere a la droga como "objeto-prótesis", al cual el sujeto recurre como respuesta a una gran carencia. Este objeto crea una falsa sensación de satisfacción, por lo que su uso se torna peligroso: "El consumidor es de hecho consumido; cree elegir, pero es elegido; piensa en actuar, pero permanece pasivo" (1992: 64).

Respecto a la edad de inicio del consumo es preocupante encontrar que muchos sujetos probaron por primera vez alguna droga entre los 10 y 14 años (más del 55\%), y que el 13,9\% lo hiciera antes de los 10 años. Según Oliver: "La totalidad de los que consumen al- 
cohol diaria o interdiariamente están ubicados en los grupos que comenzaron a beber entre los 11 y 25 años" (2004: 369). En este sentido, se podría suponer que a menor edad de inicio del consumo mayor intensidad y frecuencia de uso posterior.

Hallamos, además, que las sustancias de inicio siguen una distribución similar, en términos generales, a la que presenta la mayoría de países; es decir, un elevado consumo de drogas sociales, como el alcohol y el tabaco (Cedro, 2004). El inicio a través de drogas sociales, por la mayor parte de sujetos $(86,1 \%)$, no era de extrañar puesto que estas sustancias gozan de gran aceptación, su consumo es promovido a través de intensas campañas publicitarias en todos los medios de comunicación y reforzado por modelos de comportamiento social como son los padres, líderes y figuras del entretenimiento; son baratas y están disponibles dadas las pocas restricciones sobre su venta y comercialización.

Alarma el hecho de que estas sustancias estén al alcance de los niños desde tan corta edad, lo cual hace pensar sobre el rol que cumplen las familias, el colegio y el Estado. No cabe duda de que ante este panorama resulta necesario impulsar y desarrollar programas de prevención primaria en la población escolar.

Por otro lado, es interesante apreciar el porcentaje de adolescentes que se inician en el consumo a través de la marihuana $(7,4 \%)$, hecho que podría atribuirse a la presión social, a la necesidad de búsqueda de sensaciones y a un "afán de enfrentar o conducir sus vivencias, crisis o estados afectivos fluctuantes" (Rojas, 2005: 66).

Tomando en cuenta los resultados presentados hasta el momento, se podría decir que el alcohol y el tabaco son las sustancias de puerta de entrada al consumo de drogas ilegales. Sin embargo, también se podría afirmar, como lo hicieran Gamella y Jiménez, 2003; Rivas et al., 2000; y Zickler, 2000 (citados por Rojas, 2005), que "aquellas personas que presentaron un consumo más intenso y precoz de alcohol, tabaco y marihuana, tendrían mayores probabilidades de iniciarse en el consumo de otras drogas ilegales" (2005: 68). En efecto, encontramos que el $59,3 \%$ de sujetos desarrolló dependencia a drogas cocaínicas.

En cuanto a la prevalencia de la sustancia principal de consumo, los resultados del estudio difieren un poco de los hallados por Cedro y Rojas en los últimos años, quienes reportaron que la droga ilegal de mayor consumo era la marihuana en lugar del clorhidrato de cocaína. Sin embargo, esta diferencia se podría atribuir a que este estudio estuvo sujeto a una muestra clínica.

En lo que se refiere al consumo de drogas cocaínicas, se aprecia el fenómeno del abandono del consumo de $\mathrm{PBC}$ a favor de un mayor consumo de clorhidrato de cocaína; datos que coin- 
ciden con los encontrados por Cedro (2004). No obstante, no deja de ser significativo el porcentaje de sujetos que consumen $\mathrm{PBC}$, dadas las repercusiones de su uso a corto y largo plazo; nos referimos a la psicopatización secundaria provocada por la dependencia a la PBC que menciona Cedro (2005). Los dependientes de esta sustancia presentaron un patrón de personalidad con marcados rasgos compulsivo, antisocial, histriónico, narcisista y agresivosádico. Se podría decir que son personas ansiosas, inestables y con una marcada impulsividad, aspectos que se acentúan con el consumo compulsivo de esta sustancia.

Otro aspecto que resulta importante analizar es la prevalencia del consumo de marihuana, pues aunque en nuestra muestra se ubique en un tercer lugar, no por ello deja de ser relevante el valor alcanzado (18,5\%). El consumo de marihuana representa un riesgo para la salud, ya que a pesar de ser una droga peligrosa, el hecho de que las consecuencias más graves solo se presenten después de varios años de uso compulsivo hace que no se aprecie su peligro y se perciba como una sustancia fácil de dejar, lo cual, además, se refuerza porque muchas personas, e incluso países, estarían a favor de su uso.

La tendencia a consumir marihuana con mayor prevalencia entre los jóvenes (15 y 29 años) y aquellos con un nivel de instrucción superior, respondería posiblemente a una mayor oferta de es- ta droga en las calles y centros de estudios. A su vez, puede suponer que muchos jóvenes utilizan la droga como medio de escape para sobrellevar las presiones académicas y personales, así como también una forma de ganar prestigio entre sus compañeros. Cualesquiera de estas razones nos llevan a pensar que la estructura de personalidad de estas personas sería muy frágil, puesto que necesitan recurrir a un objeto externo (en este caso la droga) para manejar sus problemas. Los resultados respecto a la personalidad de los dependientes de esta sustancia revelan un patrón de personalidad con acentuados rasgos histriónicos, narcisistas y antisociales; además de características paranoides y compulsivas.

Respecto al consumo de alcohol, anteriormente indicamos que es la droga de inicio de mayor uso, y, además, podemos observar que se presenta como droga de consumo principal en un $15,7 \%$, especialmente entre los sujetos de mayor edad (40 a 59 años). Resulta interesante estudiar la prevalencia de esta sustancia a la luz de su rol como facilitadora en las relaciones sociales y como medio para sobrellevar una depresión latente. El hecho de que muchos adultos recurran al consumo de alcohol para desinhibirse y poder desenvolverse socialmente exhibiría una carencia de autoconfianza, autoestima y autoeficacia.

Los rasgos que definirían la personalidad del alcohólico, según los resul- 
tados hallados en el estudio, son: compulsivo, antisocial, agresivo-sádico, narcisista, esquizoide y paranoide. Estos resultados, aunque coinciden con los reportados en el estudio NESARC, discrepan de los de Craig (en Millon, 2002), que indican una prevalencia de rasgos de personalidad dependiente, evitativo, esquizotípico y límite. Por su parte, Oliver (2004), señala que el comportamiento psicopático y el trastorno antisocial de la personalidad coexisten entre los adolescentes dependientes del alcohol; aunque es más frecuente observar personalidades de tipo pasivo-dependientes.

De esta manera, queda evidenciada la relación entre los rasgos desadaptativos de personalidad y la farmacodependencia, planteada en un inicio, y la elevada prevalencia de estos rasgos entre los sujetos evaluados. En términos generales, se podría decir que la personalidad de los farmacodependientes de este estudio es similar, independientemente de la sustancia de consumo; evidenciando marcados rasgos de tipo compulsivo, antisocial, narcisista, paranoide, histriónico y agresivo-sádico. Este patrón de personalidad es bastante parecido al encontrado en el estudio NESARC, citado en Grant (2004).

\section{REFERENCIAS}

Allport, G. (1980). La personalidad. Su configuración y desarrollo. Barcelona: Herder.

American Psychiatric Association-APA (1992). DSM-III-R. Manual diagnóstico y estadístico de los trastornos mentales. Barcelona: Masson, S.A.

American Psychiatric Association-APA (1995). DSM-IV. Manual diagnóstico y estadístico de los trastornos mentales. Barcelona: Masson, S.A.

Bandura, A. (1997). Self-efficacy. The exercise of control. Nueva York: W. H. Freeman and Company.

Belloch, A. (1996). Personalidad sana y personalidad trastornada. En: A. Fierro. Manual de psicología de la personalidad. Barcelona: Paidós.

Centro de Información y Educación para la Prevención del Abuso de Drogas-Cedro (2004a). Estudio sobre drogas en universitarios de Lima. Lima: Cedro editores. [en línea]: <http://www.cedro. org.pe>.

Centro de Información y Educación para la Prevención del Abuso de Drogas-Cedro (2004b, marzo). Epidemiología de drogas en la población urbana 2003. Encuesta de hogares. Cedro: Monografía de Investigación núm. 22.

Centro de Información y Educación para la Prevención del Abuso de Drogas-Cedro (2005, junio). El problema de las drogas en el Perú. Lima: Cedro editores.

Cohen, R. y Swerdlik, M. (2001). Pruebas $y$ evaluación psicológicas. México: McGraw-Hill.

Cruz, S. (2001). Efectos y mecanismos de acción de las drogas de abuso. En: R. 
Tapia. Las adicciones: Dimensión, impacto y perspectivas. 2. ${ }^{\text {a }}$ edición. México: El Manual Moderno.

De Casarino, E., \& De Leonetti, M. (1992). Factores psicológicos tempranos en la estructuración de la personalidad adicta. En: A. Musacchio \& A. Ortiz. Drogadicción. Buenos Aires: Paidós.

Grant, B. (2004). Co-occurrence of 12month alcohol and drug abuse disorders and personality disorders in the United States. Arch Gen Psychiatry, 61: 8-361.

Hernández, R.; Fernández, C. \& Baptista, P. (2003). Metodología de la investigación. México: McGraw-Hill.

Lahuerta, A., \& T. Lloret (1994). Drogas: Sintomas y signos. Barcelona: Octaedro.

Millon, T. (1974). Psicopatología y personalidad. 2. ${ }^{a}$ edición. México: Interamericana.

Millon, T., \& Davis, R. (2000). Personality disorders in modern life. Nueva York: John Wiley \& Sons.

Millon, T. (2002). MCMI-II. Inventario clínico multiaxial de Millon-II. 3. a edición revisada. Madrid: TEA.

Minedu-Devida-ONUD-Cedro (2004). Estudio epidemiológico sobre el consumo de drogas en la población escolar de secundaria de menores. Lima: Editorial Impresos \& Diseños S.A.C.

Oficina de las Naciones Unidas de Fiscalización de Drogas y de Prevención del Delito-OFDPD (2002). Tendencias mundiales de las drogas ilícitas, 2002. Nueva York: OFDPD.

Oliver, E. (2004). Drogas: Psicopatología del uso, abuso y dependencia a las sustancias psicoactivas. Perú: Ediciones Libro Amigo.
Organización Mundial de la Salud-OMS (1992). Descripciones clínicas y pautas para el diagnóstico (CIE-10). Madrid: Meditor.

Organización Mundial de la Salud-OMS. [en línea]: <http://www.who.int/es/ index.html $>$.

Ortiz, A. (1992). Adolescencia y drogadicción. En: A. Musacchio, A. Ortiz et al. Drogadicción. Buenos Aires: Paidós.

Pelicier, Y. (1992). Dependencia y objeto totalitario. En: A. Musacchio, A. Ortiz et al. Drogadicción. Buenos Aires: Paidós.

Rojas, M. (2005). Consumo de drogas psicoactivas en un colegio de púberes $y$ adolescentes. Pautas y tendencias. Lima: Cedro/Naciones Unidas, Oficina contra la Droga y el Delito.

Sánchez-Hervás, E., y E. Berjano (1996). Características de personalidad en sujetos drogodependientes. Psicothema, 3(8), 457-463.

Sánchez-Hervás, E., Morales, E. \& Gradolí, V. (2004). Trastornos de personalidad y conductas adictivas: implicaciones en el tratamiento. Conductas Adictivas, 4(4), 1-6. [en línea]: $<$ http://www.conductasadictivas.org/co nductas/html/personalidad1.html $>$.

Tapia, R. (2001). Las adicciones: dimensión, impacto y perspectivas. 2. ${ }^{\mathrm{a}}$ edición. México: El Manual Moderno.

Villanueva, M. (1989). Factores psicológicos asociados a la iniciación y habituación. En: F. León y R. Castro de la Mata (Eds.). Pasta básica de cocaína. Un estudio multidisciplinario. Lima: Cedro.

Zavaleta, A. (2001). Factores de riesgo y protección en el consumo de drogas en la juventud. Lima: Cedro. 


\section{ANEXO}

\section{Ficha de datos personales}

La información de este cuestionario no será revelada en ningún momento. Gracias por su colaboración

Fecha de evaluación:

Nombre:

Sexo: M F Edad: Estado Civil: $\square$ Soltero $\square$ Casado $\square$ Separado / Divorciado $\square$ Viudo

Grado de instrucción:

Edad de inicio de consumo: Droga de inicio:

Droga principal de consumo: Tiempo de consumo:

Otras:

Patrón de consumo: $\square$ Experimental $\square$ Social $\square$ Habitual $\square$ Compulsivo

Tipo de tratamiento: $\square$ Ambulatorio $\square$ Internamiento $\square$ Tratamientos previos $\square$ Sin tratamiento

Tiempo de tratamiento:

Recaídas: Sí NO Medicación actual: Sí NO 